\title{
Surveillance de l'innocuité des vaccins au Canada : déclarations au SCSESSI, 2017
}

\author{
K Johnson ${ }^{1 *}, \mathrm{H}^{\text {Anyoti }}{ }^{1}, \mathrm{C}$ Coulby ${ }^{1}$
}

\section{Résumé}

Contexte : Le Canada dispose d'un système de surveillance des vaccins exhaustif qui comporte un volet de surveillance tant active que passive des vaccins administrés au Canada.

Objectifs : Fournir 1) une analyse descriptive des déclarations d'effets secondaires suivant l'immunisation (ESSI) pour les vaccins administrés au Canada, 2) un examen descriptif de I'utilisation des soins de santé après un ESSI et son issue clinique, 3) une analyse des effets secondaires graves (ESG).

Méthodologie : Les données proviennent du Système canadien de surveillance des effets secondaires suivant l'immunisation (SCSESSI), qui comprend un volet de surveillance active et passive. Nous avons effectué des analyses descriptives des déclarations d'ESSI reçues jusqu'au 30 avril 2018, en lien avec les vaccins administrés entre le $1^{\text {er }}$ janvier et le 31 décembre 2017. Les éléments de données comprenaient les ESSI, les caractéristiques démographiques, l'utilisation des soins de santé, I'issue clinique et la gravité des effets secondaires.

Résultats : Le SCSESSI a reçu 2960 déclarations d'ESSI en lien avec des vaccins administrés en 2017 dans l'ensemble du Canada. Le taux moyen annuel de déclarations d'ESSI était de 12,6 pour 100000 doses distribuées (8,1 pour 100000 habitants) au Canada pour les vaccins administrés en 2017; ce taux était inversement proportionnel à l'âge. La majorité des déclarations (91\%) portait sur des effets bénins, c'est-à-dire des éruptions cutanées au point d'injection, et des réactions allergiques. Dans l'ensemble, il y a eu 253 déclarations d'ESG, pour un taux de déclarations de 1,1 pour 100000 doses distribuées en 2017. Parmi les déclarations d'ESG, les ESSI les plus courants étaient les convulsions ( $n=58,23 \%$ ) suivies par l'anaphylaxie ( $n=33,13 \%)$. On n'a observé aucun problème inattendu d'innocuité des vaccins ni aucune augmentation de la fréquence ou de la gravité des effets secondaires attendus.

Conclusion : En 2017, la surveillance continue de l'innocuité des vaccins commercialisés au Canada n'a mis en évidence aucune augmentation de la fréquence ou de la gravité des ESSI, ni aucun ESSI jusque-là inconnu, ni aucun secteur où il aurait fallu mener des études ou des enquêtes plus approfondies. Les vaccins commercialisés au Canada continuent d'avoir un excellent profil d'innocuité.
Affiliation

${ }^{1}$ Système canadien de surveillance des effets secondaires suivant l'immunisation (SCSESSI) Centre de l'immunisation et des maladies respiratoires infectieuses, Agence de la santé publique du Canada, Ottawa (Ontario)

*Correspondance : phac.aefi-essi. aspc@canada.ca
Citation proposée : Johnson K, Anyoti H, Coulby C. Surveillance de l'innocuité des vaccins au Canada : déclarations au SCSESSI, 2017 Relevé des maladies transmissibles au Canada 2018;44(12) : 377-84. https://doi. org/10.14745/ccdr.v44i12a04f

Mots-clés : innocuité des vaccins, effets secondaires, immunisation, surveillance, SCSESSI 


\section{Introduction}

Pour détecter tout nouveau problème lié à l'innocuité des vaccins et pour maintenir la confiance du public à l'égard des vaccins, il est essentiel de surveiller l'innocuité des vaccins après leur commercialisation. L'Agence de la santé publique du Canada (ASPC) collabore avec Santé Canada, I'organisme de réglementation, pour assurer le maintien d'un système exhaustif de surveillance post-commercialisation de l'innocuité des vaccins.

Le Système canadien de surveillance des effets secondaires suivant l'immunisation (SCSESSI) est un système fédéral, provincial et territorial (FPT) de surveillance post-commercialisation de I'innocuité des vaccins. Le SCSESSI est administré par l'ASPC. II est unique dans la mesure où il comprend un volet de surveillance passive (déclarations FPT spontanées) et un volet de surveillance active. La surveillance active est assurée par le programme canadien de surveillance active de l'immunisation (IMPACT); un réseau de 12 hôpitaux pédiatriques canadiens qui scrutent les cas d'hospitalisation afin de détecter les patients ayant eu des effets secondaires suivant I'immunisation (ESSI). Les principaux objectifs du SCSESSI sont les suivants :

- Surveiller continuellement l'innocuité des vaccins commercialisés au Canada

- Détecter les hausses de fréquence ou de gravité des réactions liées aux vaccins précédemment décelées

- Cerner les ESSI jusque-là inconnus

- Déterminer les secteurs où des études et / ou des enquêtes plus approfondies s'avèrent nécessaires

- Fournir de l'information opportune sur les profils de déclaration d'effets secondaires suivant l'immunisation (ESSI) visant les vaccins commercialisés au Canada, susceptible d'éclairer les programmes et les lignes directrices en matière d'immunisation (1)

Au Canada, les fournisseurs de soins de santé, les fabricants et les membres du public ont chacun un rôle à jouer dans la vaccinovigilance (2). Les responsables FPT de la santé publique exercent une surveillance étroite de l'innocuité des vaccins par l'intermédiaire du Groupe de travail sur la vaccinovigilance (GTVV) du Comité canadien sur l'immunisation (CCI). Le GTVV réunit des représentants de tous les programmes d'immunisation à l'échelle FPT, ainsi que des autorités de réglementation de Santé Canada et d'IMPACT. Le présent rapport a été rédigé grâce à la contribution et au soutien des membres du GTVV.

Des rapports nationaux sur les données de surveillance de I'innocuité des vaccins sont publiés périodiquement $(3,4)$. Le présent rapport vise à fournir : a) une analyse descriptive des déclarations d'ESSI des vaccins administrés au Canada en 2017, b) un examen descriptif de l'utilisation des soins de santé après avoir subi des ESSI et de leur issue clinique, c) une analyse des effets secondaires graves (ESG).

\section{Méthodologie}

\section{Définitions}

Un ESSI désigne toute manifestation clinique indésirable qui suit la vaccination, sans qu'elle ait nécessairement une relation de cause à effet avec l'administration du vaccin. L'effet secondaire peut être un signe, un symptôme ou une maladie définie (5).

Dans le SCSESSI, un ESG répond à la définition de l'International Conference on Harmonization of Technical Requirements for Registration of Pharmaceuticals for Human Use, c'est-àdire qu'il s'agit d'un effet qui entraîne la mort, met la vie en danger, exige l'hospitalisation du patient ou la prolongation de son hospitalisation, entraîne une invalidité ou une incapacité persistante ou marquée, ou entraîne une invalidité ou malformation congénitale. Un événement d'ordre médical qui ne met pas la vie en danger dans l'immédiat, mais qui exige une intervention afin de prévenir l'un ou l'autre des problèmes énumérés ci-dessus peut également être considéré comme grave (6).

\section{Sources des données}

Le SCSESSI est un processus collaboratif FPT qui comprend la transmission de déclarations d'ESSI découlant de la surveillance à la fois passive et active. La surveillance passive débute à l'échelle des services de santé publique locaux. Les déclarations sont d'abord envoyées aux autorités sanitaires des provinces et territoires (PT) puis elles sont transmises sur une base volontaire à I'ASPC aux fins d'intégration au SCSESSI (7). De plus, le SCSESSI reçoit les déclarations des autorités fédérales (Services aux Autochtones Canada, Service correctionnel du Canada, la Gendarmerie royale du Canada, la Défense nationale et les Forces armées canadiennes). Ces déclarations sont saisies dans le SCSESSI et une copie de la déclaration et / ou les renseignements sur le déclarant sont envoyés aux autorités sanitaires de la province ou du territoire d'origine.

Les infirmières d'IMPACT procèdent à une surveillance active sous la supervision de médecins spécialistes des maladies pédiatriques ou infectieuses, qui étudient les admissions à I'hôpital pour relever les ESSI cibles qui auraient pu apparaître après une vaccination et qui ont entraîné une hospitalisation $(8,9)$.

Toutes les déclarations d'ESSI sont saisies dans le SCSESSI et les ESSI graves sont cernés et codés conformément au Dictionnaire médical international des activités de réglementation (MedDRA, McLean version 17, Virginie, États-Unis) (10). Des professionnels de la santé qualifiés procèdent systématiquement à l'analyse médicale des cas et déterminent la raison principale de la déclaration à l'aide des définitions nationales de cas pour la classification des ESSI, tirées du Guide de I'utilisateur du SCSESSI (11). Pour plus de renseignements sur le SCSESSI et sur le processus de déclaration et d'assurance de la qualité, veuillez consulter les déclarations déjà publiées $(3,4)$. 
Les taux de déclaration sont calculés en utilisant deux différents dénominateurs. Lorsque c'est possible, les données sur les doses de vaccin distribuées, qui sont fournies par les titulaires d'autorisation de mise sur le marché, servent à calculer le taux à partir des doses distribuées. Ce taux n'est pas corrigé en fonction des doses retournées ou gaspillées. Lorsque les données sur les doses distribuées ne sont pas disponibles, les estimations annuelles de la population de Statistique Canada servent de dénominateur pour calculer le taux en fonction de la population (12).

\section{Analyse des données}

Toutes les déclarations d'ESSI transmises au SCSESSI jusqu'au 30 avril 2018, associées à des vaccins administrés entre le $1^{\text {er }}$ janvier 2017 et le 31 décembre 2017, sont comprises dans le présent rapport. De plus, toutes les déclarations d'ESSI suivant les vaccins administrés à partir de 2007 ont été prises en considération pour évaluer les tendances sur plusieurs années. Les données ont été extraites du SCSESSI le 27 mai 2018. Remarque : I'incomplétude des déclarations transmises au SCSESSI pour 2017 est connue et s'explique par un retard de la soumission des données provenant d'une région spécifique à l'intérieur d'une province ou d'un territoire (ce qui représente moins de $2 \%$ de l'ensemble des déclarations transmises au SCSESSI en 2017).

Les analyses descriptives sont effectuées à l'aide du logiciel SAS Enterprise Guide, version 5.1 (Cary, Caroline du Nord, États-Unis) (13). Les calculs ont été présentés pour tous les vaccins combinés afin de calculer les taux globaux par nombre de doses distribuées pour l'année 2017, ainsi que les taux par année (2007-2017), le type de surveillance, la raison principale de la déclaration, les principaux ESSI selon la gravité et l'utilisation des soins de santé et les résultats cliniques pour les vaccins administrés en 2017. Les taux selon le sexe et le groupe d'âge ont été calculés en prenant comme dénominateur les estimations démographiques. Les données manquantes ont été exclues des calculs.

\section{Résultats}

Un nombre total de 2960 déclarations ont été transmises au SCSESSI par 13 provinces et territoires pour des vaccins administrés en 2017. Plus de 23 millions de doses de vaccins (par des dispensateurs de soins publics et privés) ont été distribuées, ce qui représente un taux de déclaration de 12,6 par 100000 doses distribuées. Au cours des 11 dernières années, le taux de déclaration des ESSI a diminué $(p<0,01)$ et les taux de déclaration variaient entre 12,6 et 21,9 par 100000 doses distribuées (figure 1). Dans le cas des sujets de moins de 18 ans, $7 \%(n=116)$ de l'ensemble des déclarations d'ESSI provenaient de la surveillance active. Ils représentaient $56 \%(n=116)$ de toutes les déclarations d'ESSI pour ce groupe d'âge (Note : données non présentées; les valeurs ne correspondent pas aux pourcentages car ces derniers ont été arrondis au nombre entier le plus près). La distribution est la même que par les années passées (4).

\section{Figure 1 : Nombre total de déclarations d'effets secondaires suivant l'immunisation et taux de déclaration par source et par année de 2007 à $2017^{a}$}

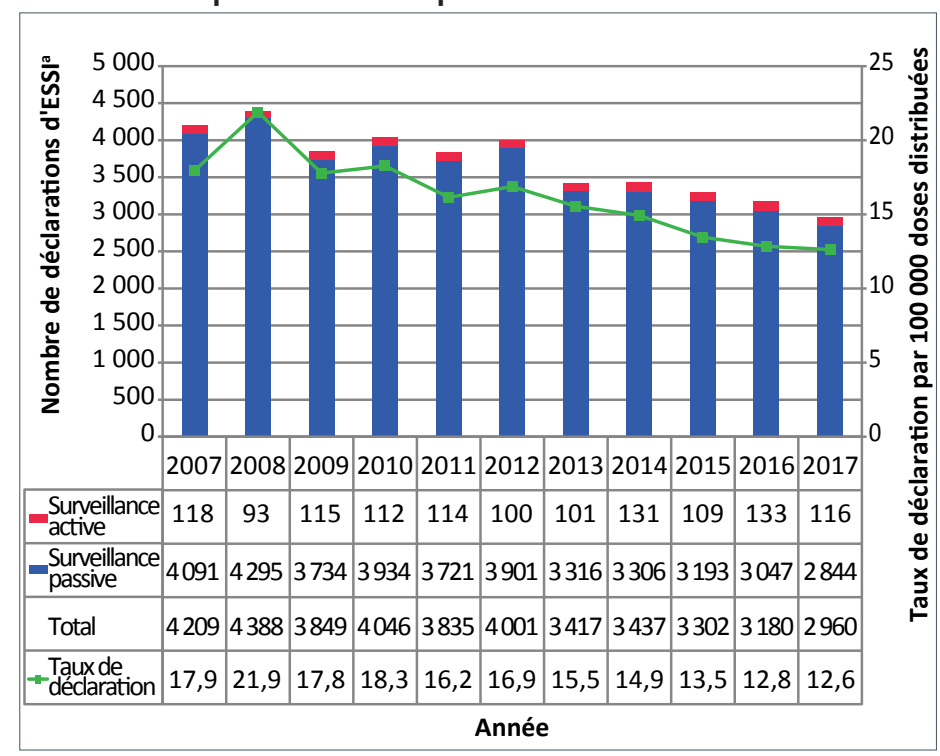

Abréviation : ESSI, effets secondaires suivant l'immunisation

a $\mathrm{N}$ inclut pas les déclarations d'ESSI suivant l'administration du vaccin contre la grippe pandémique H1N1-2009

\section{Distribution selon l'âge et le sexe}

Le nombre et les taux de déclarations pour 100000 habitants, selon le groupe d'âge et le sexe, sont présentés à la figure 2 . L'âge médian de toutes les déclarations reçues au cours de la période de déclaration était de 10 ans (étendue : âgé d'un jour à 97 ans). La majorité (60\%) des déclarations d'ESSI concernaient des enfants et des adolescents de moins de 18 ans. Le taux de déclaration le plus élevé a été observé parmi les enfants d'un an à moins de deux ans (136,5 pour 100000 habitants), suivi par les nourrissons de moins d'un an (119,6 pour 100000 habitants).

Les taux de déclaration ont diminué parmi tous les groupes d'âge de moins de sept ans $(p<0,01)$ entre 2007 et 2017, la diminution la plus importante ayant été enregistrée dans le groupe d'âge d'un an à moins de deux ans (302,5 contre 136,5 pour 100000 habitants respectivement) et le groupe des moins d'un an (182,8 contre 119,6 pour 100000 habitants respectivement) (données non présentées).

Sur 2960 déclarations, $60 \%$ concernaient des personnes de sexe féminin. Comme le montre la figure 2, une prédominance masculine a été observée chez les enfants de moins de sept ans et une prédominance féminine, chez les sujets de sept ans et plus. Les taux de déclaration des hommes et des femmes affichaient des différences importantes entre deux groupes d'âge : les adultes âgés de 18 à 64 ans avaient un ratio des taux (RT) de 4,6 (intervalle de confiance [IC] de $95 \%$; 3,86 à 5,49; $p<0,05)$ et le groupe des personnes de 65 ans et plus avait un 
RT de 2,6 (IC de $95 \% ; 2,02$ à 3,35; $p<0,05$ ), ce qui indique que les déclarations d'ESSI étaient plus susceptibles de concerner les femmes, à raison de quatre fois et demie et de deux fois et demie respectivement.

Figure 2 : Nombre de déclarations d'effets secondaires suivant l'immunisation et taux de déclaration selon le groupe d'âge et le sexe, 2017

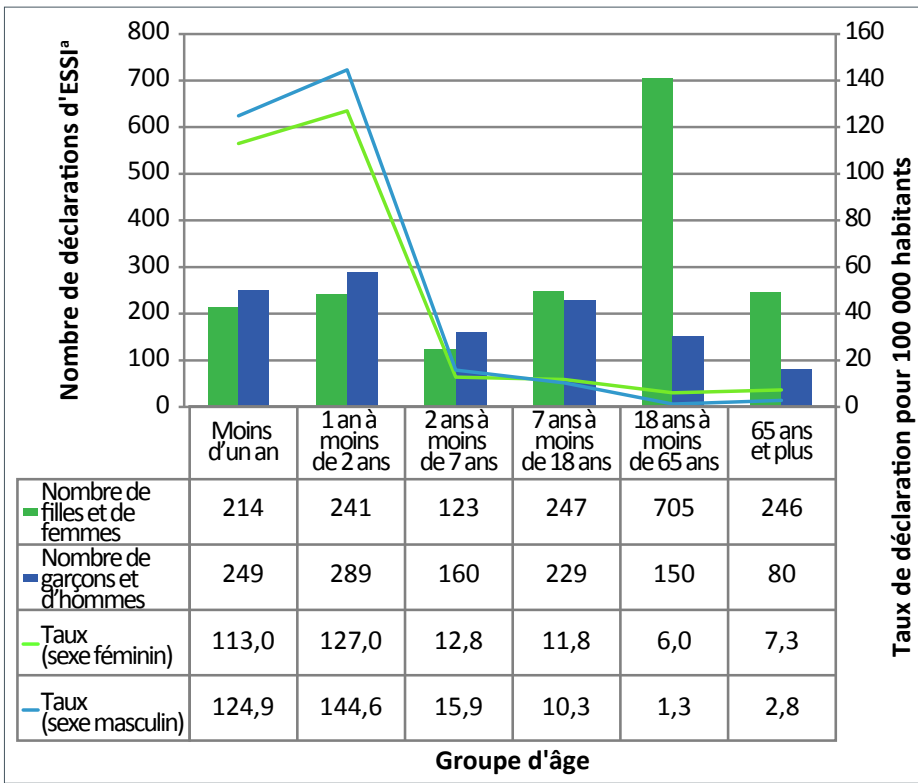

Abréviation : ESSI, effets secondaires suivant l'immunisation

a Dix-huit déclarations n'affichant pas l'âge, neuf déclarations n'affichant pas le sexe et une déclaration indiquant " autre " pour le sexe ont été exclues

\section{Principale raison de la déclaration}

Lors de l'analyse médicale des cas, la principale raison de la déclaration était est classée dans une catégorie générale d'ESSI, puis dans une sous-catégorie plus précise. À l'exception de la catégorie " autre », les principales raisons les plus courantes des déclarations pour les vaccins administrés en 2017 étaient les réactions au niveau du site de vaccination ( $n=1339,45 \%$ ) suivies des réactions allergiques ( $n=417,14 \%$ ) et des éruptions cutanées seulement ( $n=346,12 \%$ ) (tableau 1$)$.

Tableau 1 : Fréquence des déclarations et pourcentage d'effets secondaires graves pour chaque principal effet secondaire suivant la sous-catégorie de l'immunisation, 2017

\begin{tabular}{|c|c|c|c|}
\hline $\begin{array}{c}\text { Catégorie } \\
\text { principale } \\
\text { d'ESSI }\end{array}$ & $\begin{array}{c}\text { Sous-catégorie } \\
\text { de I'ESSI } \\
\text { principal }\end{array}$ & $\begin{array}{l}\text { Nombre de } \\
\text { déclarations } \\
(N=2957)^{a}\end{array}$ & $\begin{array}{c}\text { ESSI } \\
\text { graves } \\
(\%)\end{array}$ \\
\hline \multirow{5}{*}{$\begin{array}{l}\text { Réactions } \\
\text { allergiques } \\
\text { ou autres } \\
\text { manifestations } \\
\text { allergiques }\end{array}$} & Anaphylaxie & 33 & 100 \\
\hline & $\begin{array}{l}\text { Autres manifestations } \\
\text { allergiques }^{b}\end{array}$ & 355 & 1 \\
\hline & $\begin{array}{l}\text { Syndrome } \\
\text { oculorespiratoire }\end{array}$ & 28 & 0 \\
\hline & Éruption cutanée & 1 & 0 \\
\hline & TOTAL & 417 & 9 \\
\hline
\end{tabular}

Tableau 1 (suite) : Fréquence des déclarations et pourcentage d'effets secondaires graves pour chaque principal effet secondaire suivant la sous-catégorie de l'immunisation, 2017

\begin{tabular}{|c|c|c|c|}
\hline $\begin{array}{c}\text { Catégorie } \\
\text { principale } \\
\text { d'ESSI }\end{array}$ & $\begin{array}{c}\text { Sous-catégorie } \\
\text { de I'ESSI } \\
\text { principal }\end{array}$ & $\begin{array}{l}\text { Nombre de } \\
\text { déclarations } \\
(\mathrm{N}=2957)^{\mathrm{a}}\end{array}$ & $\begin{array}{c}\text { ESSI } \\
\text { graves } \\
(\%)\end{array}$ \\
\hline \multirow{7}{*}{$\begin{array}{l}\text { Infection/ } \\
\text { syndrome/ } \\
\text { symptômes } \\
\text { systémiques (ISS) }\end{array}$} & Fièvre seulement & 11 & 27 \\
\hline & Infection & 28 & 36 \\
\hline & Syndrome grippal (SG) & 10 & 0 \\
\hline & $\begin{array}{l}\text { Éruption cutanée } \\
\text { accompagnée de } \\
\text { fièvre et(ou) d'une } \\
\text { autre affection }\end{array}$ & 61 & 10 \\
\hline & $\begin{array}{l}\text { Syndromes (p. ex. } \\
\text { Kawasaki) }\end{array}$ & 16 & 88 \\
\hline & $\begin{array}{l}\text { Systémique } \\
\text { (plusieurs systèmes } \\
\text { de l'organisme sont } \\
\text { touchés) }\end{array}$ & 55 & 11 \\
\hline & TOTAL & 181 & 22 \\
\hline \multirow{9}{*}{$\begin{array}{l}\text { Manifestations } \\
\text { neurologiques }\end{array}$} & Méningite aseptique & 3 & 67 \\
\hline & Ataxie/cérébellite ${ }^{c}$ & 2 & 50 \\
\hline & Paralysie de Bell & 6 & 17 \\
\hline & $\begin{array}{l}\text { Encéphalite/ } \\
\text { encéphalomyélite } \\
\text { aiguë disséminée } \\
\text { (EMAD)/myélite }\end{array}$ & 5 & 100 \\
\hline & $\begin{array}{l}\text { Syndrome de Guillain- } \\
\text { Barré (SGB) }\end{array}$ & 2 & 50 \\
\hline & $\begin{array}{l}\text { Autre paralysie durant } \\
\text { plus d'un jour }\end{array}$ & 1 & 100 \\
\hline & Convulsions & 111 & 52 \\
\hline & $\begin{array}{l}\text { Autre manifestation } \\
\text { neurologique }^{d}\end{array}$ & 47 & 17 \\
\hline & TOTAL & 177 & 44 \\
\hline \multirow{4}{*}{$\begin{array}{l}\text { Éruption cutanée } \\
\text { seulement }\end{array}$} & Généralisée & 291 & 0 \\
\hline & Localisée & 35 & 0 \\
\hline & $\begin{array}{l}\text { Endroit non précisé/ } \\
\text { étendue inconnue }\end{array}$ & 20 & 0 \\
\hline & TOTAL & 346 & 0 \\
\hline \multirow{4}{*}{$\begin{array}{l}\text { Anxiété due à } \\
\text { l'immunisation }\end{array}$} & Présyncope & 6 & 0 \\
\hline & Syncope & 33 & 6 \\
\hline & $\begin{array}{l}\text { Autre manifestation } \\
\text { d'anxiétée }^{\text {en }}\end{array}$ & 7 & 0 \\
\hline & TOTAL & 46 & 4 \\
\hline \multirow{7}{*}{$\begin{array}{l}\text { Réactions au } \\
\text { niveau du site de } \\
\text { vaccination }\end{array}$} & $\begin{array}{l}\text { Abcès (infecté ou } \\
\text { stérile) }\end{array}$ & 13 & 31 \\
\hline & Cellulite & 329 & 5 \\
\hline & $\begin{array}{l}\text { Enflure importante } \\
\text { d'un membre }^{f}\end{array}$ & 136 & 2 \\
\hline & $\begin{array}{l}\text { Douleur au membre } \\
\text { vacciné durant sept } \\
\text { jours ou plus }\end{array}$ & 56 & 0 \\
\hline & Autre réaction locale ${ }^{g}$ & 804 & 2 \\
\hline & Éruption cutanée & 1 & 0 \\
\hline & TOTAL & 1339 & 3 \\
\hline $\begin{array}{l}\text { Erreur de } \\
\text { vaccination }\end{array}$ & $\begin{array}{l}\text { Erreur de vaccination } \\
\text { TOTAL }\end{array}$ & 3 & 0 \\
\hline
\end{tabular}


Tableau 1 (suite) : Fréquence des déclarations et pourcentage d'effets secondaires graves pour chaque principal effet secondaire suivant la sous-catégorie de l'immunisation, 2017

\begin{tabular}{|c|c|c|c|}
\hline $\begin{array}{c}\text { Catégorie } \\
\text { principale } \\
\text { d'ESSI }\end{array}$ & $\begin{array}{l}\text { Sous-catégorie } \\
\text { de l'ESSI } \\
\text { principal }\end{array}$ & $\begin{array}{l}\text { Nombre de } \\
\text { déclarations } \\
(N=2957)^{a}\end{array}$ & $\begin{array}{l}\text { ESSI } \\
\text { graves } \\
(\%)\end{array}$ \\
\hline \multirow[t]{13}{*}{ Autre } & Arthralgie & 16 & 0 \\
\hline & Arthrite & 5 & 20 \\
\hline & $\begin{array}{l}\text { Manifestation } \\
\text { gastrointestinale }\end{array}$ & 169 & 5 \\
\hline & $\begin{array}{l}\text { Épisode hypotonique- } \\
\text { hyporéactif }(E H H)\end{array}$ & 17 & 24 \\
\hline & Intussusception & 6 & 83 \\
\hline & $\begin{array}{l}\text { Anesthésie/ } \\
\text { paresthésie }\end{array}$ & 22 & 5 \\
\hline & Parotidite & 9 & 0 \\
\hline & Pleurs persistants & 16 & 6 \\
\hline & $\begin{array}{l}\text { Syndrome de mort } \\
\text { subite du nourrisson }\end{array}$ & 0 & S.O. \\
\hline & $\begin{array}{l}\text { Syndrome de la mort } \\
\text { subite }\end{array}$ & 0 & S.O. \\
\hline & Thrombocytopénie & 25 & 80 \\
\hline & Autres manifestations ${ }^{h}$ & 163 & 12 \\
\hline & TOTAL & 448 & 13 \\
\hline
\end{tabular}

Abréviations : ESSI, effets secondaires suivant l'immunisation; N, nombre total; S.O., sans objet a Trois rapports ont été exclus, car ils ne comportaient pas de sous-catégorie de l'ESSI général b La sous-catégorie « Autres » comprend, sans s'y limiter, l'hypersensibilité et l'urticaire ' L'ataxie cérébelleuse est définie par l'apparition soudaine d'une ataxie tronculaire et de troubles de la démarche (14). Notons que l'on suppose ici une absence de signes cérébelleux accompagnés d'autres signes d'encéphalite ou d'encéphalomyélite aiguë disséminée (EMAD); dans ce cas, la manifestation serait classée en fonction de la définition de cas de la Brighton dans ce cas, la manifes
Collaboration (15)

¿ La sous-catégorie "Autres » comprend, sans s'y limiter, les phénomènes convulsifs et les migraines

e La sous-catégorie « Autres » comprend, sans s'y limiter, les étourdissements et la dyspnée 'Une enflure importante d'un segment complet d'un membre proximal ou distal (ou tant proximal que distal), un « segment » étant la partie comprise entre deux articulations (16)

q La sous-catégorie "Autres » comprend, sans s'y limiter, la douleur au site de vaccination et l'enflure au site de vaccination

${ }^{h}$ La sous-catégorie «Autres » comprend, sans s'y limiter, l'adénopathie et l'arthralgie

La proportion d'effets graves était la plus élevée dans la catégorie des manifestations neurologiques (44\%), suivie par celle des infections/ syndromes/ symptômes systémiques (ISS) (22\%). Notons que les erreurs de vaccination n'apparaissent que dans un petit nombre de déclarations (moins de cinq déclarations d'ESSI) et dans aucune déclaration d'ESSI grave.

La figure 3 présente la répartition des ESSI selon la principale raison et le groupe d'âge. Les réactions au niveau du site de vaccination représentaient la majorité des ESSI dans tous les groupes d'âge, sauf chez les enfants de moins d'un an. À l'exception de la catégorie des manifestations « autres " parmi les enfants de moins d'un an, l'ESSI le plus souvent signalé était l'éruption cutanée, suivi des réactions au niveau du site de vaccination (figure 3 ).
Figure 3 : Répartition selon le groupe d'âge des principaux effets secondaires suivant l'immunisation, $2017^{a}$

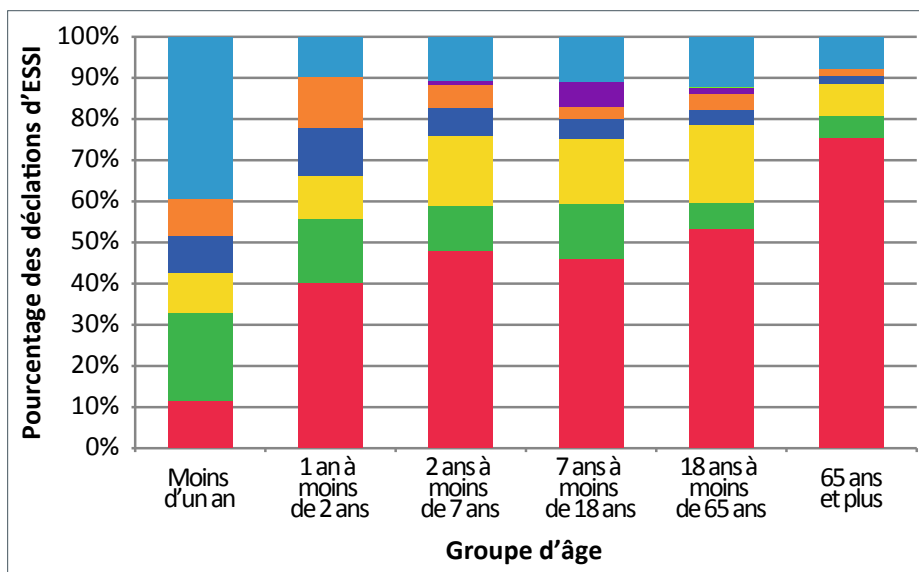
Site de vaccination
a ISS
Erreur de vaccination $\square$ Autre $^{c}$
Éruption cutanée
Réaction allergique Réaction neurologique Anxiété liée à l'immunisation

Abréviations : ESSI, effet secondaire suivant l'immunisation; ISS, Infection/ syndrome/ symptômes systémiques

a Dix-huit déclarations ont été exclues car il n'y avait aucune donnée sur les groupes d'âges, et a Dix-huit déclarations ont éte exclues car il n'y avait aucune donnée sur les grou
trois autres ont également été exclues car il y manquait les principaux ESSI

b Les ISS sont des manifestations touchant principalement plusieurs systèmes organiques, souvent accompagnées de fièvre. Ces manifestations comprennent des sous-catégories comme des syndromes reconnus (p. ex. le syndrome de Kawasaki, la fibromyalgie), la fièvre seule, le syndrome grippal et les manifestations systémiques (comme la fatigue, le malaise et la léthargie). Elles englobent également les signes d'infection d'une ou de plusieurs parties du corps

c Les autres manifestations comprennent l'arthralgie, I'arthrite, l'épisode hypotonique-hyporéactif, I'intussusception, les maladies gastro-intestinales, I'anesthésie ou la paresthésie, la parotidite, les

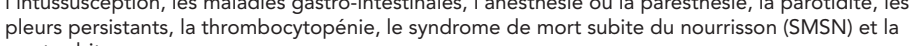
mort subite

\section{Utilisation des soins de santé}

Le tableau 2 présente le plus haut niveau de soins nécessaire suivant la manifestation d'un ESSI. L'utilisation la plus souvent signalée des soins de santé était la visite non urgente dans un établissement de soins (40\%), suivie par une visite aux soins d'urgence (24\%). La plupart des personnes faisant l'objet d'une déclaration d'ESSI (93\%) n'ont pas eu besoin d'être hospitalisées. Dans près de $23 \%$ des cas, aucun soin de santé n'a été demandé.

\section{Tableau 2 : Le plus haut niveau de soins nécessaire} suivant la manifestation d'un ESSI, 2017

\begin{tabular}{|c|c|c|}
\hline $\begin{array}{l}\text { Plus haut niveau de soins requis } \\
\qquad(\mathrm{N}=2709)^{\mathrm{a}}\end{array}$ & n & $\%$ b \\
\hline Hospitalisation requise ( 24 heures ou plus) & 197 & 7 \\
\hline $\begin{array}{l}\text { A nécessité la prolongation d'un séjour à } \\
\text { l'hôpital }\end{array}$ & 1 & $<0,1$ \\
\hline Visite au service d'urgence & 639 & 24 \\
\hline Visite non urgente & 1088 & 40 \\
\hline $\begin{array}{l}\text { Obtention de l'avis d'un professionnel de la } \\
\text { santé par téléphone }\end{array}$ & 127 & 5 \\
\hline Aucun & 623 & 23 \\
\hline Inconnu & 34 & 1 \\
\hline \multicolumn{3}{|c|}{$\begin{array}{l}\text { Abréviations : } N \text {, nombre total; n, nombre; <, inférieur à; } \\
\text { a Deux cent cinquante et un cas ont été exclus car ils ne contenaient aucun renseignement sur le } \\
\text { niveau de soins recherché } \\
\text { b Dans le tableau, le pourcentage total n'égale pas toujours } 100 \% \text { étant donné que les nombres } \\
\text { ont été arrondis }\end{array}$} \\
\hline
\end{tabular}


déclaration, cette dernière est révisée lorsque les provinces et

\section{Issue clinique}

L'issue clinique au moment de la déclaration pour l'ensemble des ESSI figure au tableau 3. Dans $75 \%$ des cas, les patients ont complètement récupéré et dans moins de $0,1 \%$, la personne est décédée. Dans les cas où la guérison est incomplète au moment de la déclaration, cette dernière est révisée lorsque les provinces et territoires transmettent l'information actualisée au SCSESSI.

Tableau 3 : Issue clinique suivant la complétion des déclarations d'effets secondaires suivant l'immunisation, 2017

\begin{tabular}{|l|r|r|}
\hline \multicolumn{1}{|c|}{\begin{tabular}{c}
\multicolumn{1}{c|}{$\begin{array}{c}\text { Issue clinique } \\
(\mathrm{N}=2878)^{\mathrm{a}}\end{array}$} \\
Guérison complète
\end{tabular}} & \multicolumn{1}{c|}{ \% } \\
\hline $\begin{array}{l}\text { La guérison n'est pas complète au moment } \\
\text { de la déclaration }\end{array}$ & 589 & 75 \\
\hline Invalidité ou incapacité permanente & 1 & 20 \\
\hline Décès & 4 & 0,1 \\
\hline Inconnu & 130 & 5 \\
\hline
\end{tabular}

Abréviations : $n$, nombre; $N$, nombre total; $<$, inférieur à

a Dans quatre-vingt-deux cas, il manquait de l'information sur l'issue clinique. Ces cas ont donc été exclus

${ }^{b}$ Dans le tableau, le pourcentage total n'égale pas toujours $100 \%$ étant donné que les nombres ont été arrondis

\section{Déclaration d'effets secondaires graves}

Sur 23 millions de doses de vaccins distribuées pendant la période de déclaration, il y a eu en tout 253 déclarations d'effets secondaires graves (ESG). Ceci représente un taux de déclarations de 1,1 pour 100000 doses distribuées et de $9 \%$ de toutes les déclarations d'ESSI en 2017. La figure 4 montre la distribution des ESG en fonction de la raison de la gravité, les cas d'hospitalisation ( $n=192)$ et les réactions mettant la vie en danger $(n=49)$ étant les raisons les plus courantes.

\section{Figure 4 : Classification des déclarations d'effets} secondaires graves, $2017^{a}$

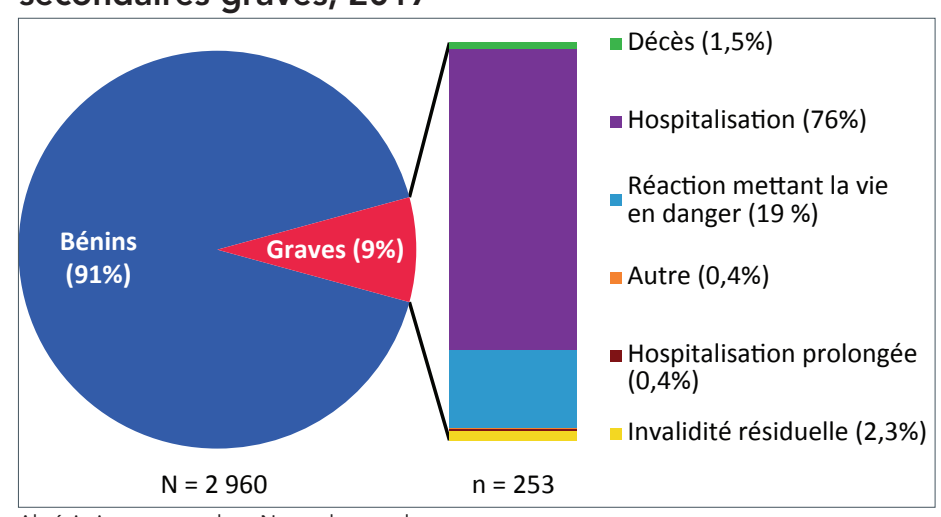

Abréviations : $n$, nombre; $N$, nombre total

a Dans la figure, le pourcentage total n'égale pas toujours $100 \%$ étant donné que les nombres ont été arrondis

Parmi les déclarations d'ESG, la réaction la plus fréquente était les convulsions ( $n=58,23 \%)$, suivie par l'anaphylaxie ( $n=33,13 \%$ ). Dans la majorité des cas d'ESG ( $n=183,72 \%)$, la guérison était complète au moment de la déclaration. Dans les cas où la guérison est incomplète au moment de la territoires transmettent l'information actualisée au SCSESSI.

Les autres issues cliniques faisant l'objet de déclarations d'ESG comprenaient les décès ( $n=4,2 \%)$, I'invalidité/incapacité permanente $(n=1,0,4 \%)$, une issue inconnue $(n=15,6 \%)$ et des données manquantes concernant l'issue $(n=5,2 \%)$.

La majorité des ESG concernent des enfants et des adolescents de moins de 18 ans ( $81 \%$ ), et près des trois quarts (74\%) de ces ESG ont été observés chez des enfants de moins de deux ans.

Il y a eu deux décès parmi les cas d'enfants de moins de deux ans et deux décès parmi les cas d'adultes de 18 ans et plus. Un examen approfondi de ces cas a montré que le décès de ces personnes était attribuable à un état pathologique préexistant (chirurgie cardiaque, blessure grave, maladie cardiovasculaire, diabète et hypertension) et non aux vaccins administrés. II y a également eu un cas d'invalidité au nombre des issues cliniques déclarées. Les antécédents médicaux de cette personne ont été passés en revue et on en est arrivé à la conclusion que l'invalidité n'était pas liée à l'immunisation.

\section{Discussion}

En 2017, le taux global de déclaration d'ESSI était de 12,6 pour 100000 doses distribuées ou de 8,1 pour 100000 habitants. Depuis 11 ans, les taux de déclaration enregistrent une tendance marquée à la baisse. Ce phénomène peut s'expliquer de plusieurs façons. Il peut être lié à une sous-déclaration, à des écarts dans la déclaration des effets attendus de nature bénigne, ou à des écarts de la couverture vaccinale.

La majorité des déclarations (91\%) concernaient des effets bénins et variaient selon l'âge. Les éruptions cutanées étaient plus fréquentes chez les nourrissons et les réactions au niveau du site de vaccination étaient plus fréquentes parmi les personnes âgées. Une prédominance masculine a été observée chez les enfants de moins de sept ans et une prédominance féminine, chez les sujets de sept ans ou plus. Ce dernier résultat est comparable à d'autres constats révélant que les femmes de la population adulte signalent invariablement plus d'effets secondaires $(3,4,17)$. Les différences observées entre les sexes en fonction de l'âge peuvent aussi être en partie expliquées par une plus importante couverture vaccinale chez les femmes (18). La majorité des ESG touchaient les enfants et les adolescents, ce qui peut en partie s'expliquer par le programme IMPACT, qui est à l'affût de cibles de surveillance précises parmi les enfants admis dans 12 hôpitaux pédiatriques de soins tertiaires $(19,20)$. Si l'on observe qu'une proportion plus importante d'enfants de moins de deux ans ont des ESSI, c'est peut-être surtout en raison du nombre de vaccins administrés aux enfants de ce groupe d'âge pour les protéger au moment où ils sont le plus vulnérables aux maladies évitables grâce à la vaccination. Bien que le pourcentage d'ESSI ait augmenté de $8 \%$ (entre 2013 et 2016) à $9 \%$ (en 2017), cette hausse s'explique peut-être par 
une diminution des déclarations d'ESSI bénins. En 2017, le taux de déclaration d'ESG correspondait aux taux des années passées et aucun problème inattendu d'innocuité des vaccins n'a été observé.

\section{Limites}

La surveillance passive des ESSI est sujette à certaines limites, comme la sous-déclaration, la sur-déclaration, l'incertitude quant à la validité du diagnostic d'un effet signalé, le manque de renseignements concernant d'autres causes possibles, comme un état pathologique sous-jacent ou des médicaments concomitants, ainsi que les différentes pratiques en matière de déclaration d'une région à l'autre du Canada.

Il existe également des limites relativement à la stratégie de surveillance active. Le programme IMPACT cible des ESSI préétablis (comme les convulsions), ce qui peut limiter la capacité de ce programme de repérer de nouvelles réactions indésirables aux vaccins. En outre, le programme IMPACT est axé sur les cas d'enfants hospitalisés, ce qui signifie que seuls les cas les plus graves sont détectés. Enfin, le programme IMPACT n'est pas exhaustif, car il ne couvre que $90 \%$ des lits et des hospitalisations pédiatriques en soins tertiaires au Canada $(19,20)$. Malgré ces limites, ce programme peut jouer un rôle important dans la surveillance de l'innocuité des vaccins, en repérant de façon active des ESSI graves ciblés dans la population pédiatrique.

De plus, on ne dispose pas du nombre de doses administrées dans la population à l'échelle nationale; par conséquent, on se sert soit des doses distribuées, soit des statistiques de la population comme dénominateur. Le meilleur dénominateur est le nombre de doses distribuées, mais il a ses limites:

- ces données ne sont pas égales au nombre de doses administrées

- $\quad$ elles ne tiennent pas compte des doses gaspillées

- le nombre de doses distribuées peut ne pas être complet au moment de la publication, en raison de retard de la part des détenteurs d'une autorisation de mise sur le marché

Pour le présent rapport, un dénominateur fondé sur la population a servi à l'analyse démographique (les taux selon le sexe et l'âge). Un dénominateur fondé sur la population suppose une distribution similaire des doses de vaccin dans les sous-groupes de la population, ce qui n'est peut-être pas systématiquement le cas. Ceci représente donc une limite de la méthode.

\section{Conclusion}

En 2017, la surveillance continue de l'innocuité des vaccins commercialisés au Canada n'a mis en évidence aucune augmentation de la fréquence ou de la gravité des ESSI, ni aucun ESSI jusque-là inconnu. La majorité des ESSI déclarés était de nature prévisible et bénigne. Les vaccins commercialisés au Canada continuent d'avoir un excellent profil d'innocuité.

\section{Déclaration des auteurs}

K. J. - Analyse officielle, validation, ébauche initiale, examen et révision

C. C. - Logiciel, analyse officielle, validation, ébauche initiale, examen et révision

H. A. - Validation, examen et révision, supervision

\section{Conflit d'intérêts}

Aucun.

\section{Remerciements}

La production du présent rapport n'aurait pas été possible sans la contribution du public, des professionnels de la santé publique, IMPACT, les enquêteurs, les infirmières qui examinent les cas, la Société canadienne de pédiatrie, et les autorités sanitaires locales, régionales, provinciales et territoriales qui ont transmis des déclarations au SCSESSI ni sans la collaboration continue des membres du Groupe de travail sur la vaccinovigilance. Nous tenons également à remercier les membres de ce groupe pour leurs commentaires et leur soutien tout au long de l'élaboration de ce rapport.

Enfin, nous remercions chaque personne qui prend le temps de soumettre une déclaration d'ESSI pour sa contribution à I'innocuité des vaccins au Canada.

\section{Financement}

Ce travail a été entièrement financé par l'Agence de la santé publique du Canada.

\section{Références}

1. Agence de la santé publique du Canada. Système canadien de surveillance des effets secondaires suivant l'immunisation. Ottawa (ON):ASPC; 2016. https://www.canada.ca/fr/ sante-publique/services/immunisation/systeme-canadiensurveillance-effets-secondaires-suivant-immunisation.html

2. Agence de la santé publique du Canada. Page 2 : Guide canadien d'immunisation: Partie 2 - Innocuité des vaccins. Ottawa (ON): ASPC; [mis à jour 2013 juin]. https://www. canada.ca/fr/sante-publique/services/publications/vie-saine/ guide-canadien-immunisation-partie-2-innocuite-vaccins/ page-2-innocuite-vaccins.html

3. Law BJ, Laflèche J, Ahmadipour N, Anyoti H. Rapport annuel du Système canadien de surveillance des effets secondaires suivant l'immunisation (SCSESSI) pour les vaccins administrés en 2012. Relevé des maladies transmissibles au Canada 2014 Déc;40 Suppl 3:7-25. DOI

4. Ahmadipour N, Watkins K, Fréchette M, Coulby C, Anyoti H, Johnson K. Surveillance de l'innocuité des vaccins au Canada 
12. Statistique Canada. Estimations de la population au 1er juillet, par âge et sexe (Tableau : 17-10-0005-01). CANSIM (database). https://www150.statcan.gc.ca/t1/tb/1/fr/tv.action ?pid $=1710000501 \&$ request_locale $=f r$

5. Council for International Organizations of Medical Sciences (CIOMS) and World Health Organization. (WHO). Definition and Application of Terms for Vaccine Pharmacovigilance. Report of ClOMS/WHO Working Group on Vaccine Pharmacovigilance. Geneva, Switzerland: CIOMS and WHO; 2012. http://www.who.int/vaccine_safety/initiative/tools/ ClOMS_report_WG_vaccine.pdf

6. International Conference on Harmonisation (ICH) of Technical Requirements for Registration of Pharmaceuticals for Human Use. ICH Harmonised Tripartite Guideline. Clinical Safety Data Management: Definitions and Standards for Expedited reporting E2A. Current Step 4 version. ICH: Oct 27, 1994. www.ich.org/fileadmin/Public_Web_Site/ICH_Products/ Guidelines/Efficacy/E2A/Step4/E2A_Guideline.pdf

7. Agence de la santé publique du Canada. Formulaire de rapport des effets secondaires suivant l'immunisation. Ottawa (ON): ASPC; 2016 Sept. https://www.canada.ca/ content/dam/phac-aspc/documents/services/immunization/ adverse-events-following-immunization-reportingdeclaration-manifestations-cliniques-inhabituelles-suiteimmunisation-fra.pdf

8. Morris R, Halperin SA, Déry P, Mills E, Lebel M, MacDonald N, Gold R, Law BJ, Jadavji T, Scheifele D, Marchessault V, Duclos P. IMPACT monitoring network: A better mousetrap. Can J Infect Dis 1993 Jul;4(4):194-5. DOI PubMed

9. Scheifele DW, Halperin SA; CPS/Health Canada, Immunization Monitoring Program, Active (IMPACT). Immunization Monitoring Program, Active: a model of active surveillance of vaccine safety. Semin Pediatr Infect Dis 2003 Jul;14(3):213-9. DOl PubMed

10. Conseil international d'harmonisation (ICH). Documents supplémentaires. Medical Dictionary for Regulatory Activities. https://www.meddra.org/how-to-use/supportdocumentation/french

11. Groupe de travail sur la vaccinovigilance et l'Agence de la santé publique du Canada. Déclaration de manifestations cliniques inhabituelles $(\mathrm{MCl})$ à la suite d'une immunisation au Canada : Guide de l'utilisateur pour remplir et soumettre les rapports de déclaration de $\mathrm{MCl}$. Ottawa (ON): ASPC; 2011 août. https://www.canada.ca/fr/sante-publique/ services/immunisation/declaration-manifestations-cliniquesinhabituelles-suite-immunisation/guide-utilisateur-remplirsoumettre-rapports-declaration-mci.html

13. SAS Enterprise Guide version 5.1. Cary, NC, USA: SAS Institute Inc., Copyright 2012. All Rights Reserved.

14. van der Maas NA, Bondt PE, de Melker H, Kemmeren JM. Acute cerebellar ataxia in the Netherlands: a study on the association with vaccinations and varicella zoster infection. Vaccine 2009 Mar;27(13):1970-3. DOI PubMed

15. Sejvar JJ, Kohl KS, Bilynsky R, Blumberg D, Cvetkovich T, Galama J, Gidudu J, Katikaneni L, Khuri-Bulos N, Oleske J, Tapiainen T, Wiznitzer M; Brighton Collaboration Encephalitis Working Group. Encephalitis, myelitis, and acute disseminated encephalomyelitis (ADEM): case definitions and guidelines for collection, analysis, and presentation of immunization safety data. Vaccine 2007 Aug;25(31):5771-92. DOl PubMed

16. Woo EJ, Burwen DR, Gatumu SN, Ball R; Vaccine Adverse Event Reporting System Working Group. Extensive limb swelling after immunization: reports to the Vaccine Adverse Event Reporting System. Clin Infect Dis 2003 Aug;37(3):3518. DOI PubMed

17. Harris T, Nair J, Fediurek J, Deeks SL. Assessment of sex-specific differences in adverse events following immunization reporting in Ontario, 2012-15. Vaccine 2017 May;35(19):2600-4. DOl PubMed

18. Agence de la santé publique du Canada. La vaccination chez les adultes canadiens : faits saillants de le 2016 Enquête nationale sur la couverture vaccinale des adultes (ENCVA). 2018. https://www.canada.ca/fr/sante-publique/services/ publications/vie-saine/2016-enquete-couverture-vaccinalenationale-adultes.html

19. Bettinger JA, Halperin SA, Vaudry W, Law BJ, Scheifele DW; Canadian IMPACT members. Le programme canadien de surveillance active de l'immunisation (IMPACT) : Surveillance active des effets secondaires suivant l'immunisation et des maladies évitables par la vaccination. Relevé des maladies transmissibles au Canada Déc;40 Suppl 3:47-50. DOI

20. Scheifele DW, Halperin SA; CPS/Health Canada, Immunization Monitoring Program, Active (IMPACT). Immunization Monitoring Program, Active: A model of active surveillance of vaccine safety. Semin Pediatr Infect Dis 2003 Jul;14(3):213-9. DOI PubMed

\section{Annexe 1 : Figures complémentaires (disponibles sur demande)}

Figure A1: Proportion des déclarations d'effets secondaires suivant l'immunisation selon la stratégie de surveillance active par rapport à la stratégie de surveillance passive parmi les enfants de moins de 18 ans, 2017

Figure A2 : Taux annuel de déclaration d'effets secondaires suivant l'immunisation selon le groupe d'âge, 2007-2017 